\title{
Intermédialités
}

Histoire et théorie des arts, des lettres et des techniques

Intermediality

History and Theory of the Arts, Literature and Technologies

\section{Différenciations romanesques}

\section{L'imaginaire technique chez Balzac, Villiers de l'Isle-Adam et Jules Verne}

\section{Étienne Beaulieu}

Numéro 6, automne 2005

URI : https://id.erudit.org/iderudit/1005510ar

DOI : https://doi.org/10.7202/1005510ar

Aller au sommaire du numéro

\section{Éditeur(s)}

Centre de recherche sur l'intermédialité

ISSN

1705-8546 (imprimé)

1920-3136 (numérique)

Découvrir la revue

Citer cet article

Beaulieu, É. (2005). Différenciations romanesques : l'imaginaire technique chez Balzac, Villiers de l'Isle-Adam et Jules Verne. Intermédialités / Intermediality,

(6), 121-139. https://doi.org/10.7202/1005510ar
Résumé de l'article

À l'ère industrielle, le roman français du XIX ${ }^{\mathrm{e}}$ siècle se donne pour tâche de créer des liens entre les différentes époques techniques par le moyen du plurilinguisme. Dans l'oeuvre de Honoré de Balzac, et en particulier dans Le chef d'oeuvre inconnu, la technique demeure encore artisanale et a fortiori les techniques artistiques (peinture, musique, sculpture), qui sont néanmoins représentées de façon à mettre en jeu la finalité de toute technè : tenter de remédier à la mort en cherchant à fixer la vie dans des traces artistiques. Dans L'Ève future de Villiers de l'Isle-Adam, la technique se transforme en technologie (et même en technomythie) par l'invention d'une femme électrique imaginée par l'ingénieur Edison. Enfin, dans Le château des Carpathes de Jules Verne, le mythe de Pygmalion se voit réécrit selon une fantasmagorie technologique qui vise encore à conserver la vie d'une cantatrice aimée, la Stilla. Dans le passage d'une époque technique à une autre, le roman français crée ainsi une continuité imaginaire par le moyen de greffes narratives et successives qui mettent en jeu la différenciation romanesque.
Ce document est protégé par la loi sur le droit d'auteur. L'utilisation des services d'Érudit (y compris la reproduction) est assujettie à sa politique d'utilisation que vous pouvez consulter en ligne.

https://apropos.erudit.org/fr/usagers/politique-dutilisation/ 


\title{
Différenciations romanesques
}

\author{
L'imaginaire technique chez Balzac, \\ Villiers de I'Isle-Adam et Jules Verne
}

\author{
Étienne Beaulieu
}

L

e régime d'historicité ${ }^{1}$ moderne, s'interprétant lui-même selon la ligne de

partage de l'Être et de l'Autre, semble n'avoir compris la technique qu'en regard de ces notions, sans égard à ce qui en elle fait pourtant trembler une frontière appelée à s'effacer, sinon à se déplacer sur un terrain où ce départage ne serait plus aussi étanche. Afin de contrecarrer l'oubli de l'Être, les uns font en

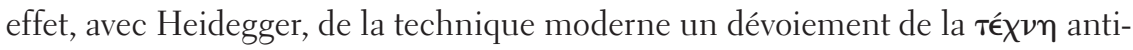
$q^{2}$. Tandis que d'autres, avec Lévinas par exemple, ramènent la question de la

1. Je reprends ce terme à François Hartog, qui l'entend ainsi, après Hegel, Heidegger, Gadamer et Ricœur: "l'expérience d'une distance de soi avec soi, que je nomme rencontre avec l'historicité.» (François Hartog, Régimes d'historicité. Présentisme et expériences du temps, Paris, Éditions du Seuil, coll. «La librairie du XXI ${ }^{\mathrm{e}}$ siècle, 2003, p. 64) Il n'est pas certain cependant que cette scission ne soit pas ce qui, précisément, fonde la séparation moderne du Même et de l'Autre par laquelle historicité et Modernité deviennent pratiquement synonymes, par exemple chez Hegel, dans son partage entre sociétés historiques et sociétés sans histoire (voir Georg W. F. Hegel, La raison dans l'histoire, trad. Kostas Papaioannou, Paris, Union générale d'éditions, 1955 [1828], en particulier «Permanence et historicité», p. 205-208). C'est en effet, dans la perspective hégélienne, l'appel de la liberté qui détermine un peuple à se saisir historiquement lui-même et à s'arracher à ses déterminations afin de se faire autre. La notion d'«historicité» a donc elle-même une racine historique moderne.

2. Est-il besoin d'insister cependant sur le contresens que l'on fait souvent subir au texte de Heidegger en lui prêtant l'intention de diaboliser la technique? Il s'agit pour le philosophe de considérer ce qui «dans la technique est essentiel, au lieu de nous laisser fasciner par les choses techniques» (Martin Heidegger, «La question de la technique», dans Essais et conférences, trad. André Préau, Paris, Éditions Gallimard, coll. «Tel», 1958 [1954], p. 44). Rappelons seulement que Heidegger rejette l'alternative «qui nous forcerait à nous jeter tête baissée dans la technique ou, ce qui reviendrait au même, à nous révolter inutilement contre elle et à la condamner comme œuvre diabolique. » (p. 34) 
technique au jeu du battement entre Être et Autre en lui découvrant le pouvoir de «supprimer le privilège de l'enracinement [...] dans le Lieu » et d'ouvrir ainsi au visage et à l'altérité. Dans ce débat, il n'est au fond pas tellement question de technique que d'ontologie ou de son dépassement. C'est sur le terrain de la technique que l'ontologie débat avec elle-même, sans s'aviser toutefois du glissement qui se produit si l'on déplace l'enjeu sur la technique elle-même et que se pose la question de ce qu'elle est en regard d'un changement de paradigme qui ferait passer de l'Autre à la différence. Ce qui se produit alors consiste non plus à considérer la technique en regard de l'ontologique, où elle est forcément seconde et instrumentale, mais à faire passer la technique au premier rang et à examiner l'ontologique sous l'angle de la technique, c'est-à-dire qu'il s'agit de penser ce qui est habituellement second comme quelque chose de premier, de faire de ce qui vient après ce par quoi tout débute.

Une relecture de l'histoire de l'ontologie pourrait en effet découvrir, au lieu d'une ontologie de la technique, une ontologie technique ${ }^{4}$ qui ferait de cette

3. Emmanuel Lévinas, Difficile liberté, Paris, Albin Michel, coll. «Présences du judaïsme », 2003 [1963].

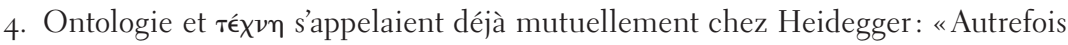

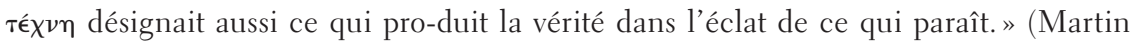
Heidegger, «La question de la technique», p. 46) Il est vrai qu'il s'agit pour le philosophe du sens «humble» de la technique, qui, dans l'Antiquité, se groupait avec «l'art» sous ce nom, avant que la «nouvelle conception de la technique» (Paolo Rossi, Les philosophes et les machines, 1400-1700, trad. Patrick Vighetti, Paris, Presses universitaires de France, 1996, p. 5) ne sépare nettement art et technique, le premier se rangeant du côté du savoir

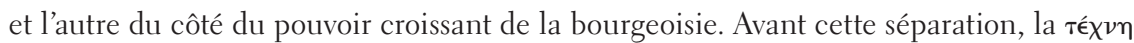
permettait ce que Heidegger appelle dans Être et Temps (selon la traduction byzantine de Vezin), un « déloignement» (Être et Temps, trad. François Vezin, Paris, Éditions Gallimard, 1986 [1927], p. 145), c'est-à-dire une forme «artisanale» de rapprochement de l'Être. Dans la mesure où c'est l'essence de la technique que nous gardons en vue, il n'est pas impossible d'effectuer ce rapprochement en regard de la technique moderne, mais à partir d'une ontologie d'emblée et non accessoirement technique. C'est par exemple à cette utopie que pense Habermas lorsqu'il enjoint à la réflexion sur «la reprise de [la] technique au sein du monde vécu de la pratique» (Jürgen Habermas, La technique et la science comme «idéologie», trad. Jean-René Ladmiral, Paris, Éditions Gallimard, coll. «Essais », 1973 [1968], p. 87; c'est l'auteur qui souligne). Plus près de nous, c'est à ce projet que se consacre Marc Desportes en s'attachant au «paysage d'une technique [qui] se forme selon un processus ouvert, influencé par la technique elle-même». La perception du paysage tient donc sa source de l'histoire de la technique, par exemple les vues aériennes de la terre donnent naissance, au début du $\mathrm{xx}^{\mathrm{e}}$ siècle, à une autre façon d'habiter et de 
dernière un médium par lequel serait disloqué le rapport des moyens et des fins 5 . Le «moyen sans $f_{i n}{ }^{6}$ » de l'ontologie technique permettrait de faire passer la relation entre un moyen et la fin visée, d'ordinaire seconde, à l'avant-plan: la relation deviendrait alors première sur la substance au cœur de l'ontologique, maintenant conçu lui-même comme une technique, un médium. Dans l'ontologie platonicienne, les quatre segments de l'Être (eikasia [copie], pistis [créance], dianoia [discursion], noèsis [intellection]7), importeraient moins en eux-mêmes que dans la relation entre les dimensions de l'Être qu'elles définissent par le principe platonicien de l'analogie, qui est non seulement un rapport essentiel entre des segments de l'Être, une relation primordiale, mais surtout rapport de rapports. De même, dans la distribution des catégories de l'Être chez Aristote (substance, quantité, qualité, relation, lieu, temps, position, possession, action, passion), ce ne serait pas tant la substance globale de l'Être que la relation des catégories entre elles qui formerait la condition de possibilité de toute vérité ${ }^{8}$. L'Être, chez Aristote, se présenterait comme une substance faite de qualité, quantité, etc., mais une substance échappant elle-même à sa substantialité première (substantialité d'étant, dira Heidegger), qui relie un substrat à un prédicat dans

concevoir l'espace. Examinant la technique moderne sous cet angle, Desportes retrouve Heidegger et «la question primordiale [qui] concerne la façon dont l'homme habite le monde» (Marc Desportes, Paysages en mouvement. Transports et perception de l'espace $\mathrm{XVIII}^{e}-\mathrm{XX}^{e}$ siècle, Paris, Éditions Gallimard, 2005, p. 11-12). Ontologie et technique, loin de s'exclure, sont intrinsèquement liées, même dans la technique moderne.

5. C'était déjà ce à quoi Heidegger invitait lorsqu'il cherchait à mettre en question le préjugé de la technique comme instrument: «Aussi longtemps que nous nous représentons la technique comme un instrument, nous restons pris dans la volonté de la maîtriser. Nous passons à côté de l'essence de la technique.» (Martin Heidegger, «La question de la technique », p. 44)

6. Pour Giorgio Agamben, la «forme de vie» est par exemple un moyen sans fin, puisque «une vie qui ne peut être séparée de sa forme est une vie pour laquelle, dans sa manière de vivre, il y va de la vie même et, dans son vivre, de son mode de vie. » (Giorgio Agamben, Moyens sans fins, Paris, Éditions Payot, coll. «Bibliothèque Rivages», 2002 [1995], p. 14)

7. Platon, République, VI, 510-511.

8. Ce qu'Aristote indique d'ailleurs lui-même: «Aucun de ces termes en lui-même et par lui-même n’affirme ni ne nie rien ; c'est seulement par la liaison de ces termes entre eux que se produit l'affirmation ou la négation. (Aristote, «Catégories », Organon, trad. Jean Tricot, Paris, Librairie philosophique Vrin, 1959, p. 6, 2a 4-5) Ce premier niveau de la liaison produit aussi bien une syllogistique qu’une rhétorique ou encore une métaphysique attributive. 
un premier temps, pour se chercher, dans un deuxième temps, comme être en tant qu'être, c'est-à-dire comme relation pure considérée en tant que telle dans sa différence ontologique9 ${ }^{9}$ Dans la mesure où la relation serait considérée comme sa dimension première, l'ontologie se découvrirait ainsi l'aspect technique d'un appareil ${ }^{10}$ servant à fabriquer de la différence et conduirait à la mise au jour d'une intermédialité ontologique de laquelle découleraient les rapports entre les différents médias. La technique ne serait ainsi plus seconde, ni première d'ailleurs, puisque c'est par elle qu'ontologiquement cette distinction se trouverait prise dans une différence qui la précéderait.

\section{LA TECHNIQUE ROMANESQUE}

I24 Se contentant de tracer ce programme (par définition inachevable, puisque la relation considérée en elle-même, demeurant en puissance faute de la délimitation nette d'une relation en acte, s'ouvre sur une infinité de relations possibles), il convient par contre de donner un exemple, qui sera ici celui de l'appareil romanesque moderne considéré comme une autre forme de fabrique technique de la différence. Afin d'entrer dans cet imaginaire technique du roman, par lequel le genre romanesque s'imagine lui-même comme une technique, suivons la pensée du narrateur de La recherche au moment où il se rend pour la première fois à Balbec en train.

Les levers de soleil sont un accompagnement des longs voyages en chemin de fer, comme les œufs durs, les journaux illustrés, les jeux de cartes, les rivières où des barques s'évertuent sans avancer. À un moment où je dénombrais les pensées qui avaient rempli mon esprit pendant les minutes précédentes, pour me rendre compte si je venais ou non de dormir (et où l'incertitude même qui me faisait me poser la question était en train de me fournir une réponse affirmative), dans le carreau de la fenêtre, au-dessus d'un petit bois noir, je vis des nuages échancrés dont le doux

9. Il ne s'agit pas ici d'interpréter Aristote en fonction de l'analogie platonicienne, qui ferait de l'Être une théorie de la participation analogique du particulier au général, où l'Être serait le genre supérieur de tous les genres. Ce n'est donc ni de l'analogie de proportionnalité (platonicienne), ni de l'analogie d'attribution (que discute Pierre Aubenque dans Le problème de l'être chez Aristote, Paris, Presses universitaires de France, 2002 [1962], p. 202) dont il est question, mais de l'Être en tant qu'être comme relation prise comme homonyme à elle-même.

10. J'entends ce terme au sens où Jean-Louis Déotte (L'époque des appareils, Paris, Lignes Manifestes, 2004) lui donne sens, par exemple dans sa caractérisation de la cure analytique comme appareil. Ce n'est en effet pas seulement les appareils «matériels» qui informent le réel, mais aussi les appareils conceptuels, du roman jusqu'à l'ontologie elle-même. 
duvet était d'un rose fixé, mort, qui ne changera plus, comme celui qui teint les plumes de l'aile qui l'a assimilé ou le pastel sur lequel l'a déposé la fantaisie du peintre. Mais je sentais qu'au contraire cette couleur n'était ni inertie, ni caprice, mais nécessité et vie. Bientôt s'amoncelèrent derrière elle des réserves de lumières. Elle s'aviva, le ciel devint d'un incarnat que je tâchais, en collant mes yeux à la vitre, de mieux voir, car je le sentais en rapport avec l'existence profonde de la nature, mais la ligne du chemin de fer ayant changé de direction, le train tourna, la scène matinale fut remplacée dans le cadre de la fenêtre par un village nocturne aux toits bleus de clair de lune, avec un lavoir encrassé de la nacre opaline de la nuit, sous un ciel semé de toutes ses étoiles, et je me désolais d'avoir perdu ma bande de ciel rose quand je l'aperçus de nouveau, mais rouge cette fois, dans la fenêtre d'en face qu'elle abandonna à un deuxième coude de la voie ferrée; si bien que je passais mon temps à courir d'une fenêtre à l'autre pour rapprocher, pour rentoiler les fragments intermittents et opposites de mon beau matin écarlate et versatile et en avoir une vue totale et un tableau continu ${ }^{11}$.

Cet extrait renvoie à l'ensemble de La recherche en tant qu'il met en scène le passage entre un état et un autre, de Paris à Balbec, entre sommeil et veille, entre nuit et jour, au seuil vitré du dedans et du dehors. Dans cette brèche temporelle et spatiale, qui d'un côté laisse voir la rougeur du soleil levant et de l'autre le bleu de la nuit finissante, le train s'avance comme la technique elle-même qui rompt l'homogénéité d'un premier état pour en faire entrevoir un second. Le paysage matinal a perdu la pureté du premier regard jeté sur lui par le narrateur et doit être recomposé, ses fragments doivent être «rentoilés» pour reconquérir leur totalité, mais une totalité imaginée. À l'intérieur de cette brèche, le narrateur tente de refaire un monde, un tout, avec la brisure introduite par le tournant du train. Ce tournant figure le roman lui-même, en tant qu'il brise un état pour en créer un autre, le perd pour mieux le retrouver, et parce qu'il pave la voie à la transformation de la vie ancienne du narrateur (son enfance parisienne) en une nouvelle vie, en sursis, à créer, à rentoiler. Lobjet technique qu'est le chemin de fer n'apparaît pas ici par hasard: Proust recueille une tradition littéraire de «paysages ferroviaires ${ }^{12}$ » commencée au XIX ${ }^{\mathrm{e}}$ siècle et poursuivie par delà La modification de Michel Butor. Le train est en quelque sorte la technique qui a brisé l'unité du paysage et forcé le romancier à en créer un autre parce qu'il entraîne une situation inédite, un lieu de pure relation situé entre tous les états, qui permet

11. Marcel Proust, À l'ombre des jeunes filles en fleurs, Paris, Éditions Gallimard, 1954 [1913], p. 240.

12. Marc Desportes, «Les paysages artificiels. Les chemins de fer 1830-1860», dans Paysages en mouvement, p. 99-156. 
cette transformation d'une culture première en une culture seconde, pour reprendre les termes du philosophe Fernand Dumont ${ }^{13}$. Inversement, la brèche s'ouvrant entre un monde au départ entier et le nouveau monde à recomposer se découvre comme technique romanesque, c'est-à-dire comme topos d'une vie rompue, fragmentée et recomposée, «stylisée » selon les mots de Dumont, par une médiation technique. Les illusions qui se perdent dans le roman dévoilent ainsi les limites d'un premier monde et, traçant cette limite technique, en ouvrent un second qui est et n'est pas le premier, puisqu'il le recompose mais le fait exister seulement dans une image où il se présente en différé, dans un autre temps et un autre lieu.

C'est le rapport entre cette image de la vie recomposée et la vie elle-même qui est mise en scène dans les trois romans ici pris à témoin (Le chef-d'œeuvre inconnu, L'Ève future, Le château des Carpathes). Dans ces trois romans, une femme est transformée en image grâce à des moyens techniques de plus en plus sophistiqués, qui demeurent simplement artistiques, au sens traditionnel (et heideggerien) chez Balzac, et deviennent technologiques, voire techno-mythiques (et lévinassiens) chez Villiers de l'Isle-Adam et Verne. Dans ce parcours cependant, le relais entre les techniques artistiques et les technologies imaginaires ou romanesques s'effectue dans une continuité qui laisse envisager un même projet de conserver ou de préserver la vie par le moyen de la technique qui aboutit chez Jules Verne à une forme de survie technologique. Technique et technologie renvoient de ce point de vue l'une à l'autre, comme le simple et le complexe demeurent de même nature, mais différent en degré.

Plus encore, l'on pourrait dire que la fixation de la vie dans des images correspond aux différenciations s'effectuant pour Bergson dans la manifestation de l'élan vital qui, dans son actualisation, laisse derrière lui les scories de sa différenciation. Ces images de la vie, comme autant de stases de la succession des instants dans la durée, semblent secondaires ou frappées d'une presque inexistence par rapport à la durée elle-même puisqu'elles n'en sont que de simples coupes temporelles. Mais, comme le rappelle Deleuze, «ces fictions ne sont pas de simples hypothèses: elles consistent à pousser au-delà de l'expérience une direction prélevée sur l'expérience elle-même ${ }^{14}$ ». Prélevées sur l'expérience mais la prolongeant en dehors de ses conditions normales d'existence, les images techniques de la vie dans ces trois romans miment la différenciation de la durée.

13. Fernand Dumont, Le lieu de l'homme. La culture comme distance et comme mémoire, Montréal, Les éditions Hurtubise HMH, 1968.

14. Gilles Deleuze, Le bergsonisme, Paris, Presses universitaires de France, 2004 [1966], p. 16. 
Entre la vie et la fixation dans son image, les frontières se déplacent: la vie est conservée dans une peinture dans Le chef-d'ouvre inconnu, aussi existe-t-elle avant l'image; elle est enregistrée à l'intérieur du corps de la créature artificielle d'Edison dans L'Ève future et elle y a donc une existence simultanée à celle de son image - elle en est la synthèse, une vie synthétique; tandis que la vie tente de se poursuivre par-delà sa propre image dans Le château des Carpathes.

\section{L'INCONNUE DU CHEF D'đUVRE}

Dans Le chef-d'œuvre inconnu, la technique trouve dans le plurilinguisme romanesque un moyen d'expression privilégié qui se répercute sur le genre même du roman et affecte sa présentation de lui-même. En plusieurs lieux de son œuvre, Balzac laisse se refléter l'ensemble de La comédie humaine, à commencer par le personnage de Vautrin, qui parle le langage de tous les milieux, comme le tissu textuel qui lui donne naissance. La cathédrale fournit de même, selon la célèbre métaphore proustienne, une image de cette œuvre dans laquelle on pénètre par des entrées différentes pour découvrir chaque fois des perspectives nouvelles. Moins célèbre, mais néanmoins révélateur, l'instrument de musique forgé par le compositeur Gambara, dans la nouvelle éponyme, donne à lire une image de l'ensemble de la Comédie humaine en ce que ce fameux instrument, nommé le «panharmonicon», peut « remplacer un orchestre entier ${ }^{15} »$. Cet «instrument aux cent voix» (G, p. 116) est «aussi grand qu'un piano à queue, mais ayant un buffet supérieur de plus. Cet instrument bizarre offr[e], outre ce buffet et sa table, les pavillons de quelques instruments à vent et les becs aigus de quelques tuyaux $^{16}$ ». (G, p. 115) Sorte d'ancêtre du synthétiseur, le panharmonicon, figure de l'instrument romanesque balzacien, est un assemblage technique qui vise à remplacer tous les autres langages instrumentaux et à réunir toutes les voix dissemblables dans une harmonie aussi vibrante et réelle que «la lumière, [qui] est décomposée par nos arts comme le rayon par le prisme». (G, p. 115) Mais, précisément, le panharmonicon souffre de n'être qu'un bricolage technique, puisque «l'état imparfait dans lequel se trouvait cette singulière machine arrê-

15. Honoré de Balzac, Gambara, introduction, notes et documents par Marc Eigeldinger et Max Milner, Paris, Éditions Flammarion, 1981 [1839], p. 115. Désormais, les références à cet ouvrage seront indiquées par le sigle « $\mathrm{G}$ » suivi de la page et placées entre parenthèses dans le corps du texte.

16. Cet instrument, comme le soulignent Eigeldinger et Milner, «a été inventé par le mécanicien allemand Léonard Maelzel, né à Ratisbonne en 1783 et mort à Vienne en 1855. » (G, p. 258) 
tait les développements du compositeur, dont la pensée parut alors plus grande ». (G, p. 116) La «folie » de Gambara consiste en ce que sa pensée dépasse le moyen purement technique de son instrument et de façon d'autant plus flagrante que le panharmonicon a pour but de recréer l'harmonie des arts musicaux. Qui veut faire l'ange fait la bête, c'est-à-dire, dans le langage de l'époque romantique: qui veut recréer la totalité au moyen de la technique risque de mettre au jour une œuvre fragmentaire, qui n'aura de vie que celle du monstre mis au jour par Frankenstein, l'être fantastique du roman de Marie Shelley (1817), dont le rapiècement artificiel ne saurait longtemps survivre, puisque si les hommes possèdent le pouvoir prométhéen de la technique, le secret de la création demeure malgré tout sous la garde de la réserve divine. En regard de cette recréation technique ou verbale, c'est donc une musique angélique et silencieuse qui se laisse entendre aux auditeurs de Gambara, mais derrière le brouhaha du panharmonicon.

Il s'agit de même dans Le chef-d'ouvre inconnu de «dérober le secret de $\operatorname{Dieu}^{17}$ » grâce à la technique artistique, c'est-à-dire de percer le voile des apparences pour arriver à rendre en peinture «l'âme» de l'objet représenté. Aussi la question est-elle de distinguer entre la poétique de l'imitation et celle, plus conforme au romantisme, de l'expression : «La mission de l'art n'est pas de copier la nature, mais de l'exprimer! Tu n'es pas un vil copiste, mais un poète!» (CDI, p. 43), s'exclame le vieux peintre Frenhofer. "Poète », c'est-à-dire, dans un premier sens (aristotélicien), un façonneur, un technicien (Frenhofer est présenté par Balzac comme l'héritier du «faire » [CDI, p. 54] de Mabuse), mais aussi, en un autre sens, une sorte de guetteur patient du moment où l'âme se montre à travers la matière: «La beauté est une chose sévère et difficile qui ne se laisse point atteindre ainsi : il faut attendre des heures, l'épier, la presser, l'enlacer étroitement pour la forcer à se rendre». (CDI, p. 44) Pratique amoureuse et tâtonnante, qui, en cherchant à enserrer l'âme, se change en une rhétorique de séduction de la forme, l'art de Frenhofer se donne pour but de prêter vie à la matière. Elle n'est donc pas qu'une affaire de technique. Ou plutôt, la technique n'en est qu'une approche. Approche de quoi? De ce rien qui apparaît grâce à la technique, mais ne saurait s'y réduire: «C'est cela, et ce n'est pas cela. Qu'y manque-t-il? Un rien, mais ce rien est tout». (CDI, p. 45) Sans cette attente amoureuse de l'âme, qui distingue le génie du vulgaire, l'art n'est pour Frenhofer qu'une simple technique qui ne peut

17. Honoré de Balzac, Le chef-d'œuvre inconnu, édition présentée et annotée par Adrien Goetz, Paris, Gallimard, 1994 [1831], p. 41. Désormais, les références à cet ouvrage seront indiquées par le sigle «CDI» suivi de la page et placées entre parenthèses dans le corps du texte. 
mettre au jour que des fantômes: «Vous avez l'apparence de la vie, mais vous n'exprimez pas son trop-plein qui déborde, ce je ne sais quoi qui est l'âme peutêtre et qui flotte nuageusement sur l'enveloppe». (CDI, p. 45) Que la technique ne puisse qu'engendrer l'apparence de la vie, cela demeurera un topos critique qui perdurera jusqu'aux premières descriptions du cinématographe ${ }^{18}$, c'est-à-dire au moment où la technique a déjà bien entamé sa mutation en technologie.

Mais au-delà de la vague évocation de l'âme, qu'est-ce que ce «rien»? Albert Béguin remarquait à propos de ce passage que «toute la métaphysique balzacienne de l'énergie, de l'inépuisable force vitale, origine commune de la matière et de l'esprit, est sous-entendue dans ce discours d'esthétique exaltée ${ }^{19}$ ». «Énergie», «force vitale», c'est-à-dire, dans le langage balzacien, l'électricité. L'on se souvient que l'avant-propos de La comédie humaine, rédigé en 1842 pour l'édition Furne, identifie explicitement la "pensée à un fluide » qui avait trouvé sa formulation quelques années plus tôt dans Louis Lambert (1832), notamment dans le premier fragment post-mortem retrouvé dans les papiers du personnage éponyme: «Ici-bas, tout est le produit d'une substance éthérée, base commune de plusieurs phénomènes connus sous les noms impropres d'Électricité, Chaleur, Lumière, Fluide galvanique, etc. ${ }^{20}$ ». Durant la même période, quelques années après l'invention de la pile par Volta, Hegel, de même, fait de l'électricité l'exemple parfait d'une négativité qui se surmonte dialectiquement elle-même, dans le rapport entre les courants positifs et négatifs, pour créer une nouvelle positivité $^{21}$. Cette idée d'une électricité vitale est reprise par Balzac, qui fait de Louis

18. Par exemple, Maxime Gorki, comparant, dès 1896, le cinématographe au «royaume des Ombres et des spectres » (cité dans Emmanuelle Toulet, Le cinématographe: invention du siècle, Paris, Éditions Gallimard, Réunion des Musées nationaux, 1988, p. 135).

19. AlbertBéguin, Balzac luetrelu, Paris, Éditions du Seuil, coll. «Langages », 1965, p. 231.

20. Honoré de Balzac, Louis Lambert, préface de Raymond Abellio, Paris, Livre de poche, 1968 [1832], p. 166.

21. «L'électricité elle-même n'est pas la différence en soi, ou dans son essence ne se trouve pas la double essence de l'électricité positive et négative. C'est pourquoi on dit ordinairement qu'elle $a$ la loi d'être de cette façon, ou bien encore qu'elle a la propriété de s'extérioriser ainsi. [...] Cette indifférence prend une autre forme quand on dit que la définition de l'électricité implique que l'électricité est comme positive et négative, ou que c'est là uniquement son concept et son essence. Alors son être désignerait son existence en général [...]» (Georg W. F. Hegel, La phénoménologie de l'esprit, trad. Jean Hyppolite, Paris, Aubier, 1941 [1807], p. 126-127; c'est l'auteur qui souligne). Existant comme constituée de positif et de négatif, l'électricité a comme concept de surmonter sa nature contradictoire. Elle est l'exemple parfait d'une dialectique en acte dans le plein sens du terme. 
Lambert le héros génial de la passivité électrique, créateur passif de ce que le courant actif de l'électricité nerveuse (et animale ${ }^{22}$ ) laisse imprimer en lui. Louis Lambert est en quelque sorte le prototype de l'esthétique romanesque moderne, l'ancêtre de la passivité sans mesure à laquelle aspire Maurice Blanchot ${ }^{23}$, qui fait de la création une passivité active ou une activité passive du génie 24 . Avec l'avènement de la démocratie, cette poétique du génie passif-actif s'étend aux masses, qui deviennent, par exemple dans Notre-Dame de Paris de Victor Hugo, les figures anonymes d'un drame s'écrivant lui-même. Se découvrant indissociable de l'idée de génie rabattue sur celle de peuple, comme le rend encore plus évident la mise en cause contemporaine de cette notion au nom de celle de multitude qui l'avait précédée 25 , le roman moderne suppose un circuit populaire dans lequel le singulier et le collectif puissent se surmonter l'un l'autre, comme le positif et le négatif de l'électricité. Se serait ainsi déroulé sur le plan de l'esthétique romanesque le remplacement de la notion de multitude par celle de peuple, qui irait de pair avec celle de roman moderne, qui ne peut s'installer, bien avant l'arrivée de l'électricité « réelle», que dans le circuit de l'électricité imaginaire et populaire mis en place avec et par le régime esthétique et romanesque.

Sur le plan de la technique artistique mise en scène dans Le chef-d'ouvre inconnu, il s'agirait, autrement dit, si l'on se permet de rabattre le mysticisme de Louis Lambert sur la perspective picturale du peintre Frenhofer, de «faire passer » la «substance électrique» (ou galvanique) dans une forme, de lui donner un corps technique et artistique afin d'animer, au sens fort, ce fantôme formel. Mais dans la quête d'équilibre entre l'âme (électrique) et la technique picturale, c'est la seconde qui l'emporte dans le tableau «Catherine Lescaut» dont Frenhofer voudrait, en bon prométhéen de l'univers balzacien, qu'il «ne soit pas une créature, mais une création. » (CDI, p. 6o) Étonné, le jeune Poussin qui assiste à la leçon du maître, «ne voit là que des couleurs confusément amassées et contenues par une multitude de lignes bizarres qui forment une muraille de

22. Hegel est aussi, ne l'oublions pas, l'auteur d'un traité portant sur le magnétisme animal.

23. «La passivité est sans mesure.» (Maurice Blanchot, L'écriture du désastre, Paris, Éditions Gallimard, 1980, p. 34)

24. C'est ce régime de l'art que Rancière a nommé «régime esthétique » en tant qu'il permet et valorise, à travers la singularité des produits artistiques contre les règles classiques, «l'identité fondamentale des contraires. " (Jacques Rancière, Le partage du sensible. Esthétique et politique, Paris, La Fabrique, 2000, p. 33)

25. Paolo Virno, Grammaire de la multitude. Pour une analyse des formes de vie contemporaine, trad. Véronique Dassas, Paris, L'éclat, Montréal, Conjonctures, 2002. 
peinture. » (CDI, p. 66) Bien plus qu'une préfiguration visionnaire de l'art abstrait, cette description d'une toile réduite à ses éléments purement formels évoque dans l'univers balzacien l'échec de l'artiste à faire passer la vie dans la forme. La femme-tableau Catherine Lescaut, que Frenhofer rêve en femme "réelle ${ }^{26}$ ", cette femme-tableau, donc, redevient, lorsque présentée à des regards extérieurs, un simple tableau sans plus de vie qu'un amalgame de matériaux techniquement agencés. Aussi bien dire que cet échec rend explicite l'impossibilité, continuellement éprouvée par les héros balzaciens, de se substituer à Dieu par les moyens techniques de l'art. Cet échec dénonce aussi le «réalisme» de Balzac, qui, bien plus que par son réalisme de forme, pense avec toute l'époque romantique la technique comme venant après l'Être et devant donc, en tant que simple moyen, s'estomper face à sa finalité.

\section{UNE FEMME ÉLECTRIQUE}

Cette substitution demeure tout aussi impossible dans L'Ève future de Villiers de l'Isle-Adam, mais la tentative prométhéenne s'y complexifie d'un degré supplémentaire. Selon son amant, nommé Lord Ewald, Miss Alicia Clary peut être comparée à "une Déesse bourgeoise 27 », ce qui constitue, pour une conscience «artiste» du XIx ${ }^{\mathrm{e}}$ siècle, l'oxymore absolu. Figure inverse du Quasimodo hugolien, qui est une belle âme enfermée dans un corps hideux, Alicia Clary est une bourgeoise sotte et convenue enfermée dans un corps de déesse que Lord Ewald consent à prendre pour modèle d'une Ėve nouvelle, qui n'est autre qu'une femme électrique fabriquée de toutes pièces par l'ingénieur Edison. À plusieurs reprises, Lord Ewald se désespère en comparant le corps d'Alicia Clary à la Vénus victrix du Louvre, comme si la seule ressemblance de forme d'une femme et d'une sculpture, donc d'un être vivant et d'une ouvre d'art (comme dans Le chef-d'œuvre inconnu), ne suffisait pas à sa soif d'idéal. À la technique artistique, Edison fait place, avec l'accord de Lord Ewald, à la technologie (mythique) que permet d'imaginer la découverte de l'électricité.

Le problème en est donc un, encore tout romantique, de non-coïncidence du fond et de la forme et de non-concordance entre la répétition du même et

26. «Ma peinture, ce n'est pas une peinture, c'est [...] une femme avec laquelle je pleure, je ris, je cause et pense. » (CDI, p. 60)

27. Villiers de l'Isle-Adam, L'Ève future [1886], dans (Euvres complètes, édition établie par Alan Raitt et Pierre-Georges Castex, Paris, Éditions Gallimard, coll. «Bibliothèque de la Pléiade », 1986, p. 804. Désormais, les références à cet ouvrage seront indiquées par le sigle «EF » suivi de la page et placées entre parenthèses dans le corps du texte. 
la différence. Villiers avait déjà traité ce problème sur le mode ironique dans ses Contes cruels, notamment dans «Le plus beau dîner du monde », où il est question d'un dîner parfaitement réussi que défie cependant l'un des convives en en organisant un plus beau l'année suivante. La surprise des invités de ce dîner répété est de découvrir exactement le même menu que lors du premier dîner, mais de se surprendre à juger que le second dîner est «le même... et, cependant, plus beau! » (EF, p. 656) À la fois même et différent se découvre aussi l'automate fabriqué par Edison, au point où, renouvelant l'exploit technique du peintre Zeuxis dont les raisins peints trompaient même les oiseaux, Lord Ewald s'y trompe lui-même et prend la réalisation technologique de l'ingénieur pour la sotte Alicia Clary, jusqu'à ce que la créature électrique lui parle avec l'accent des plus sublimes poètes du siècle et que Lord Ewald en tombe amoureux, croyant retrouver enfin son amour pour Alicia Clary.

Comment une créature électrique peut-elle réciter des répliques apparemment improvisées? La réponse (imaginaire) à cette question plonge dans les sources mythiques de la création de Villiers. Pour cet aristocrate né en un siècle qu'il jugeait immonde, l'essentiel est de garder son génie au-dessus de la masse vulgaire, c'est-à-dire qu'il s'agit pour lui de ne pas répéter dans son œuvre l'automatisme de la conversation des gens ordinaires mise en conserve dans le sottisier de Flaubert. Ou du moins, il s'agit, s'il y est contraint, de répéter en y ajoutant ce rien qui fait tout et qui transforme un automatisme en œuvre d'art, comme le voulait le vieux Frenhofer. Se laissant porter par les mots, Villiers donne corps à cet automatisme dans l'automate fabriqué par Edison qu'il dote cependant d'un héritage conversationnel gravé sur un cylindre par la technique, toute nouvelle à l'époque, du phonographe. Bien plus qu'une femme électrique, l'Ève future est une femme-livre, puisque sa conscience «électro-magnétique » (EF, p. 830) est le produit d'un amalgame de citations que le phonographe a transformées en voix écrites mais néanmoins parlées. Presque tous les commentateurs de Villiers ont insisté sur la dimension "parlée» de son écriture, dont les tournures, souvent abruptes, ne sont pas dépourvues d'effets de voix ${ }^{28}$. Et nombre de contemporains de Villiers ont assuré l'avoir entendu «improviser» l'un de ses récits avant d'en figer la vibration vocale dans l'écriture. Ce qui signifie que Villiers transporte dans L'Ève future la question obsessive de son œuvre, qui concerne la fixation de la parole vive et sa résurrection par l'écrit. Toutes les technologies dont se sert Edison pour faire «naître » son automate découlent de l'utilisation de l'électricité

28. Pour une synthèse éclairante de cette question: Anne Le Feuvre, Une poétique de la récitation: Villiers de l'Isle-Adam, Paris, Éditions Honoré Champion, 1999. 
à des fins scripturaires: phonographe, photographe, photochromie et photosculpture procèdent en effet de la fixation de la vie par la graphie et font remonter l'écriture à l'origine de toute technè ${ }^{29}$. Mieux, elles font remonter la technè à l'origine même de la parole, puisque le rêve qui donne lieu à toute cette fantasmagorie est celui qu'Edison prononce à voix haute : «inventer un instrument qui répète avant même qu’on ait parlé». (EF, p. 772) Répétition qui précède le répété, technique qui donne lieu à l'improvisation, ce rêve n'est autre que l'automate personnifié auquel succombe l'amour de Lord Ewald $3^{\circ}$.

L'ordre des choses bascule donc de Balzac à Villiers, puisque si dans l'œuvre du premier la technique suivait la vie, l'imitait jusqu'à chercher à en reproduire l'âme, dans l'œuvre du second, la technique, par un surcroît imaginaire, vient se loger au cour de la vie : la technique devient l'âme du vivant, sa mémoire graphique et archivistique (archè : fondement). La « résurrection» de la parole par l'écrit s'accomplit ainsi dans l'acte de lecture ou d'audition, comme Edison invite Lord Ewald à y consentir lorsqu'il l'enjoint à « prêter son âme » au mécanisme des citations de la voix phonographique de sa créature électrique. C'est là l'illusionnisme de Villiers, pour qui rien n'est réel que ce à quoi nous consentons en nous aidant des traces techniques que laissent ici-bas nos existences. Monde peuplé d'ombres et de fantômes, mais non pas de fantoches, comme les distinguait Jacques Noiray, pour qui « le fantoche, c'est la machine réduite à son mécanisme pur, à la somme des pièces qui le composent. Le fantôme, au contraire, c'est la machine poétique-

29. Les métaphores de la lecture, de l'impression et de la correction d'épreuves reviennent assez fréquemment pour rendre compte du fonctionnement de cette voix automatisée; par exemple: «Maintenant, je lis les gestes sur ce Cylindre aussi couramment qu'un prote lit à rebours une page de fonte (question d'habitude): je corrigerai, disonsnous, cette épreuve selon les mobilités de Miss Alicia Clary. (EF, p. 912; c'est l'auteur qui souligne)

30. L'automate, parce qu'il rend possible la cohabitation des contraires (il est et n'est pas celui qu'il représente, dans une inquiétante étrangeté dont a su tirer parti la littérature depuis Hoffmann), est une créature esthétique dans laquelle les contraires cohabitent. L'automate offre simultanément une présence et une absence de l'image à elle-même, une «ressemblance cadavérique » dira Blanchot (L'espace littéraire, Paris, Éditions Gallimard, 1955, p. 344), aussi est-ce par le moyen du personnage de l'automate que le cinéma, comme le roman du XIX ${ }^{\mathrm{e}}$ siècle, effectuera une brisure entre l'image-mouvement et l'image-temps, selon les termes de Deleuze, puisque «l'automate est coupé du monde extérieur, mais il y a un dehors plus profond qui vient l'animer. » (Gilles Deleuze, Cinéma 2. L'image-temps, Paris, Éditions de Minuit, coll. «Critique», 1985, p. 233) Lautomate est en ce sens un habitant de la brisure temporelle et de la brèche technique dans laquelle se rompt l'illusion référentielle en s'y confondant par ressemblance. 
ment transfigurée ${ }^{31} »$. C'est, pourrait-on ajouter, la machine rêvée autant que la machine rêvante du roman.

La machine première de l'univers de Villiers, c'est bien sûr la machine romanesque elle-même, que doit transfigurer la poésie du lecteur. Mais il s'agit du roman comme machine non pas seulement au sens d'une «technique romanesque », mais surtout en regard de la multiplication des langages au sein d'un même univers qui donne lieu à son plurilinguisme. Dans une scène tirée des premières versions de L'Ève future, des oiseaux mécaniques, sortis du cerveau de l'Edison romanesque de Villiers, viennent prononcer de confuses paroles autour de Lord Ewald:

Ce fut, d'abord, UNE HUPPE BLEUE DE L'HUDSON, qui s'écria, tout à coup, avec la voix d'un jeune clergyman, et comme s'empressant avec une sollicitude suppliante: «N'accède pas à de tels vœux!... ». Balancé sur la fausse fleur séculaire d'un cactus, UN OISEAU-MOUCHE, l'interrompit, d'un accent étonné, en regardant le jeune homme: «Pourquoi se troubler de la sorte?» [...] UN BEAU CYGNE, entre deux gorgées de neige, tournant son col dédaigneux, leur notifia d'une voix de jeune miss glaciale: «À vous taire, je vous exhorte! » Ici, L'OISEAU DE PARADIS, de la voix d'un jeune élégant de l'Union [...] «Sommes-nous ses amis, messieurs?» À cette interpellation et comme pénétrés d'une déférence particulière, TOUS LES OISEAUX, se tournant vers lui, répondirent avec des voix rudes et franches: «Oui! » (Ce « oui » fut terrifiant: on eût dit qu'une soixantaine d'individus hurlaient dans un club $[\ldots])$. (EF, p. 1602-1603) $)^{32}$

Étonnante chorégraphie hallucinée d'oiseaux parleurs, qui reprennent sur tous les tons des morceaux de langage qu'ils semblent avoir amassé ici et là comme autant de brindilles. Cette variante rappelle La tentation de saint Antoine, dont Flaubert faisait paraître la dernière version dans les mêmes années (1874): dans ces deux œuvres, les discours s'objectivent et s'enfoncent dans une distance étrange qui leur confère un aspect théâtral, mécanique et automatisé, comme s'ils étaient prononcés par des bouches en délire ne s'adressant à personne et demeurant aussi impersonnelles que des machines. Machines textuelles, ces oiseaux n'ont pas la responsabilité de parole que Platon, dans sa méfiance, exprimée dans le Phèdre, à l'égard de la peinture et de l'écriture, souhaitait à l'emploi du verbe - aussi parlent-elles à tort et à travers, ne s'adressant à personne et répétant un discours sans consistance.

31. Jacques Noiray, Le romancier et la machine. L'image de la machine dans le roman français (1850-1900), Paris, Les éditions José Corti, 1982, p. 329.

32. Villiers de l'Isle-Adam, Euvres complètes, p. 1602-1603. 
Mais il s'agit aussi dans ces deux œuvres de percer à jour les voiles de la technique romanesque. À la différence du saint de Flaubert, qui peut faire un signe de croix à la fin de sa nuit de délire et se recueillir dans un silence divin, le Lord Ewald de Villiers est averti par l'automate dont il est amoureux que s'il cherche à comprendre le mécanisme de sa vie artificielle, «Dieu s'en retirera » (EF, p. 873) et que se fera entendre par sa bouche synthétique, le chant frêle d'une humanité sans Dieu, comme ce chant de rossignol "phonographié» en donne l'image acoustique toute grésillante. Car, précisément, le rossignol qu'entend Lord Ewald est «mort depuis des mois» (EF, p. 874); il ne demeure de lui que le corps glorieux de son artifice. Derrière la technique, il n'y a rien, que la mort, qui n'est «rien » que pour une humanité s'étant acquitté de sa dette envers son créateur et dont les hommes se déclarent «quittes» (EF, p. 998) de la vie entre eux, comme Edison, sauvé jadis par l'argent de Lord Ewald et, inversement, comme Lord Ewald sauvé du suicide par l'expédient technique d'Edison.

Entre ces hommes sans Dieu, il n'y a plus que l'échange technique d'une femme artificielle, qui est l'image de toutes les femmes, puisque, selon le vieux topos misogyne, «toute femme tient plus ou moins d'une andréide» (EF, p. 905) en ce qu'elle a «beaucoup d'électricité en elle.» (EF, p. 843) Mais l'andréide a ceci de pratique qu'il n'y a nul besoin d'argumenter avec elle, d'abuser de sophismes et de rhétorique pour la contraindre à sa volonté, puisqu'il suffit, pour lui faire exécuter quoi que ce soit, de simplement appuyer sur la bague appropriée. Ou encore, comme le dit Edison, l'andréide a encore ceci de charmant que «même après l'œuvre accomplie [...], vous pouvez toujours la détruire, la noyer, si bon vous semble, sans déranger pour cela le Déluge.» (EF, p. 844) La femme artificielle revêt ainsi chez Villiers l'idée de l'artifice éternel de la femme, cette «jolie créature voilée, dont le néant est sympathique» (EF, p. 849) et qui pour cela devient le support et le prétexte à une rêverie technique. Puisque la femme comme la technique ont été créées en second, selon la mythologie traditionnelle, l'une de la côte d'Adam et l'autre du feu dérobé à Zeus par Prométhée, la logique de l'histoire les propulse toutes deux au premier plan lorsque, à l'époque industrielle où est écrite L'Ève future, la Tradition bascule et que s'annoncent des temps nouveaux dans lesquels la répétition sera première.

\section{SURVIES TECHNOLOGIQUES}

«Au commencement était la répétition » ferait un bon exergue à l'œuvre de Jules Verne, puisqu'il y est souvent question de rapports entre une culture première et 
une culture seconde33 où le récit progresse de façon à montrer que sous les apparences d'une culture première, souvent rurale, traditionnelle ou tout simplement conventionnelle, se loge une culture seconde plus « originelle» qui n'est accessible que grâce à des moyens techniques qui font tout le fantastique des Voyages extraordinaires. C'est par exemple le monde paléolithique que découvre, dans Voyage au centre de la terre, l'expédition du professeur Lidenbrock, aussi bien grâce au guide Hans qu'aux ingénieux appareils de Ruhmkorff. Ce monde ancien conservé sous la croûte terrestre où s'agite le XIX ${ }^{\mathrm{e}}$ siècle est une certaine image du paléolithique ressuscité, mais pour y accéder, la technique demeure indispensable, aussi bien la technique ancienne des boussoles, gnomon et autres instruments millénaires, que la technique moderne et tous ses appareils dérivés de l'électricité, comme la lampe artificielle qui éclaire les souterrains labyrinthiques creusés par le temps et la lave dans les massifs du Sneffels. De même, dans Vingt mille lieues sous les mers, se découvre aux passagers du Nautilus l'univers sans âge du monde sous-marin grâce à la machine de théâtre qu'est le submersible imaginé par Verne, ou encore l'expédition lunaire, dans De la terre à la lune, permet aux membres du Gun club de sortir de la stratosphère (presque cent ans avant Gagarine, dont Lévinas fera l'homme habitant hors du Lieu) et d'atteindre l'orbite lunaire par le moyen d'un gigantesque canon imaginaire qui les y propulse.

Il est significatif que, dans chacun de ces récits, les voyageurs techniques aient à passer par une réduction de leur existence et par une survie qui frôle bien souvent la mort: survie souterraine au cœur de la noirceur dans Voyage au centre de la terre, survie presque coupée de tout contact avec la surface terrestre dans Vingt mille lieues sous les mers, survie à une existence limitée au minimum vital dans De la terre à la lune (mais surtout dans sa suite, Autour de la lune). Pour bien marquer le point, puisque tout, ou presque, est explicite chez Verne, le mystérieux capitaine Nemo se déclare en effet mort à lui-même, «aussi bien mort que ceux [...] qui reposent six pieds sous terre 34 !». Nul besoin d'insister sur le nom que lui choisit Verne, dont la signification est «personne» et fait implicitement référence à l'impersonnalité d'Ulysse dans l'épisode du cyclope Polyphème de cette ancienne épopée de la mer qu'est L'odyssée. Les personnages de Verne sont en effet le plus souvent des individus morts à eux-mêmes, des survivants technologiques délivrés de leurs devoirs terrestres, mais qui pour cela redécouvrent avec

33. Selon les termes du philosophe Fernand Dumont, qu'il explique notamment dans son ouvrage Le lieu de l'homme, p. 41.

34. Jules Verne, Vingt mille lieues sous les mers, préface de Christian Chelebourg, Paris, Librairie Générale française, 1990 [1870], p. 132. 
un regard nouveau l'espace et le temps. La littérature vernienne est ainsi une littérature pour enfants seulement si l'on insiste sur ce qui dans l'expression tient de la finalité: elle est une littérature pour l'enfance, c'est-à-dire en vue de l'enfance, écrite dans le but de redonner, par-delà la technique romanesque, une nouvelle virginité au temps et à l'espace. Si bien que l'œuvre de Verne offre l'image du roman comme du récit de l'homme rendu à lui-même après son existence technique. C'est l'homme revenu à une culture première par la voie improbable d'une culture seconde poussée à son excès. En cela, et beaucoup plus qu'en regard de sa croyance au progrès, Jules Verne participe pleinement de l'héritage des Lumières, qui, comme le notait Jean Starobinski35, pourrait se résumer dans le mythe de Pygmalion, puisqu'il s'agit le plus souvent d'y retrouver la simplicité de l'immanence au bout de la transcendance de la technique.

Mais c'est dans Le château des Carpathes que se donne le plus clairement à lire une réécriture technologique du mythe de Pygmalion qui embrasse les questions liées à la survie technologique d'une culture ancienne maintenue en vie au sein d'un monde nouveau. Dans le petit village transylvain de Werst, les habitants sont terrifiés par différents phénomènes provenant du château, apparemment abandonné et situé à quelques lieues de là. Après une expédition infructueuse au château, les phénomènes inexplicables se produisent à nouveau et se multiplient: apparition d'éclairs aux couleurs surnaturelles, voix d'outre-tombe surgissant au beau milieu de l'auberge du village, etc. Tout ceci demeure inexpliqué jusqu'à ce que le comte Franz de Télek, qui passait là par hasard, découvre le subterfuge technique imaginé par le Baron de Gortz, revenu habiter le château pour y conserver jalousement l'image et la voix de la Stilla, cantatrice dont il était amoureux et qui était promise à Franz de Télek avant qu'elle ne meure sur une scène d'Italie.

Les phénomènes demeurés inexplicables aux habitants de Werst sont en fait des manifestations provenant des appareils issus du travail de «l'ingénieur » Orfanik, bras droit du baron de Gortz, qui a utilisé la ressource technique traditionnelle du roman au XIX ${ }^{\mathrm{e}}$ siècle: l'électricité, que Jules Verne, après Balzac et Villiers, appelle «l'âme de l'univers ${ }^{36}$ ». Entre autres appareils que «l'illustre Edison et ses disciples avaient parachevé» (CC, p. 199), on retrouve dans Le châ-

35. Voir l'analyse du Pygmalion de Rousseau dans Jean Starobinski, Jean-Jacques Rousseau. La transparence et l'obstacle, Paris, Éditions Gallimard, 1971, p. 90-92.

36. Jules Verne, Le château des Carpathes, postface de Jean-Louis Leutrat, Paris, Actes Sud, 1997 [1892], p. 199. Désormais, les références à cet ouvrage seront indiquées par le sigle «CC » suivi de la page et placées entre parenthèses dans le corps du texte. 
teau des Carpathes, le téléphone, le téléphote - qui permet de «se voir à distance dans des glaces reliés par des fils, grâce à l'invention du téléphone» (CC, p. 200] - , le phonographe, qui a servi à enregistrer le chant de la Stilla, et un appareil optique dépourvu de nom qui permet de faire apparaître la Stilla en images37. Bref, il s'agit là de tout l'arsenal d'appareils romanesques destinés à recréer la présence humaine à partir, encore une fois, des traces de l'existence d'une femme dont les médiations traditionnelles des techniques artistiques ne suffisent plus à satisfaire l'adoration du Baron de Gortz. Toutes ces techniques artistiques sont en effet frappées de vétusté, que ce soit la poésie - le narrateur tente vainement de rendre compte de la voix de la Stilla par un vers de Musset: «...cette voix du coeur qui seule au cour arrive» (cC, p. 134) - ou encore la peinture (le Baron de Gortz «possédait le plus beau des portraits que le grand peintre Michel Gregorio eût fait de la cantatrice» (CC, p. 137), sans que cela le soulage de son deuil). C'est que la technologie mythique à laquelle fait appel Jules Verne par le truchement du personnage d'Orfanik, rassemble les mêmes finalités mais supplante en efficacité ces techniques artistiques «anciennes ». Par exemple, le fameux appareil optique qui sert à donner une image de la Stilla reproduit dans son jeu de glaces inclinées, le portrait de Gregorio qui «la représentait en pied avec son costume blanc de l'Angelica d'Orlando». (cC, p. 223) C'est-à-dire que le nouvel appareil imaginaire ne peut exister, même dans le roman, qu'en venant se greffer à l'appareil perspectiviste et pictural du tableau ${ }^{3}$. Ce qui donne au roman la tâche de secréter des liens culturels et imaginaires entre les différentes époques techniques ou technologiques et nous permet de penser, comme le souligne Déotte, que «les époques ne se suivent pas, l'une chassant l'autre: l'invention d'un appareil procède plutôt par différenciation 39 ». Le roman serait, dans l'ordre de l'imaginaire technique, le lieu de cette différenciation, au sens bergsonien du terme dans la mesure où la vie fixée dans des images se détache chaque fois d'elle-même pour engendrer une nouvelle image, coupe immobile prélevée à même le mouvement qui donnera lieu, quelques années après Le château des Carpathes, à «l'invention » du cinéma dans lequel la coupe immobile de mouvement deviendra mobile.

37. «Or, au moyen de glaces inclinées suivant un certain angle calculé par Orfanik, lorsqu'un foyer puissant éclairait ce portrait placé devant un miroir, la Stilla apparaissait, par réflexion, aussi "réelle" que lorsqu’elle était pleine de vie et dans toute la splendeur de sa beauté.» (CC, p. 223)

38. Voir Jean-Louis Déotte, L'époque de l'appareil perspectif: Brunelleschi, Machiavel, Descartes, Paris, Éditions de L'Harmattan, 2001.

39. Jean-Louis Déotte, L'époque des appareils, p. 50. 
Au carrefour des époques et des techniques, la prose romanesque aurait ainsi la fonction de liant imaginaire, du moins au XIX ${ }^{\mathrm{e}}$ siècle, où les ruptures historiques ont été orchestrées de pair avec les ruptures techniques de l'ère industrielle. Si le roman du XIX ${ }^{\mathrm{e}}$ siècle a tâché de lier l'imaginaire des techniques artistiques à celui des technologies nouvelles liées à l'électricité (phonographe, téléphone, photographie), ce même roman lègue au $\mathrm{xx}^{\mathrm{e}}$ siècle la tâche de faire le pont entre ces techniques, devenues à leur tour «traditionnelles », et la nouveauté du monde cinématographique (qui s’annonçait déjà par bribes, notamment chez Villiers ou Robida ${ }^{40}$ ), ou encore de déplacer la question vers ce que Lyotard nommera «les immatériaux » et ce qui sera connu bientôt comme «réalité virtuelle».

40. Par exemple: «La vision, chair transparente, miraculeusement photochromée, dansait en costume pailleté, une sorte de danse mexicaine populaire. Les mouvements s'accusaient avec le fondu de la Vie elle-même, grâce aux procédés de la photographie successive, qui, le long d'un ruban de six coudées, peut saisir dix minutes des mouvements d'un être sur des verres microscopiques, reflétés ensuite par un puissant lampascope.» (EF, p. 897) et Albert Robida, Le vingtième siècle: la vie électrique, Genève, Slatkine reprints, 1981 [1893]. 